\title{
Mitral valve repair versus replacement in patients with ischemic mitral regurgitation
}

\author{
Meng-Lin Lee ${ }^{1,2}$, Thay-Hsiung Chen ${ }^{1}$, Hsien-Da Huang ${ }^{2}$, Shaw-Min Hou ${ }^{1}$ \\ ${ }^{1}$ Division of Cardiovascular surgery, Department of Surgery, Cathay General Hospital, Taipei; ${ }^{2}$ Institute of Bioinformatics and Systems Biology, \\ Department of Biological Science and Technology, National Chiao Tung University, Hsinchu \\ Contributions: (I) Conception and design: ML Lee, SM Hou; (II) Administrative support: SM Hou; (III) Provision of study materials or patients: TH \\ Chen, SM Hou; (IV) Collection and assembly of data: ML Lee; (V) Data analysis and interpretation: ML Lee; (VI) Manuscript writing: All authors; \\ (VII) Final approval of manuscript: All authors. \\ Correspondence to: Meng-Lin Lee, MD. Division of Cardiovascular Surgery, Department of Surgery, Cathay General Hospital, No.280, Sec. 4, Ren Ai \\ Rd., Taipei. Email: martin25_lee@hotmail.com.
}

Background: The optimal management of ischemic mitral regurgitation (IMR) is controversial. The aim of this study was to examine our eight years' experience of surgical treatment in patients with IMR, and to compare outcomes of mitral valve repair versus replacement with concomitant coronary artery bypass grafting (CABG).

Methods: A retrospective, observational, cohort study was undertaken to collect data on consecutive patients with IMR and coronary artery disease who received CABG and mitral valve surgery in our hospital between January 2008 and December 2015. Basic patient characteristics, operative data, and postoperative clinical outcomes were examined.

Results: The series included 22 consecutive patients (21 male; 1 female). The mean age was $62.1 \pm 11.4$ years old. The mean preoperative left ventricular ejection fraction (LVEF) was $33.4 \% \pm 15.4 \%$. The mean cardiopulmonary bypass $(\mathrm{CPB})$ time was $165.4 \pm 38.4$ minutes, and the mean aortic cross clamp time was $113.8 \pm 33.6$ minutes. Eighteen patients underwent CABG plus mitral valve repair, and four patients underwent CABG plus mitral valve replacement (MVR). There were three early in-hospital mortalities: two in the mitral valve repair group, and one in the replacement group. The follow-up was complete in all patients, with a mean follow-up duration of $3.1 \pm 2.3$ years. The mean last LVEF was $35.3 \% \pm 17.7 \%$. There were 2 late mortalities. Both were from the repair group. The overall late survival rate was $81.6 \%$, with $83.0 \%$ in the repair group and $75.0 \%$ in the replacement group. In patients with echocardiography follow-up of more than or equal to 1 year duration, the residual or recurrent mitral regurgitation rates were $0.0 \%$ in the replacement group and $57.1 \%$ in the repair group. One patient in the repair group later underwent MVR due to severe regurgitation postoperatively.

Conclusions: Our preliminary findings showed that the surgical outcome of mitral valve repair might be comparable to that of MVR in terms of early mortality and long-term survival. However, mitral valve repair was associated with a higher residual or recurrent mitral regurgitation rate. According to the latest literature, the role of MVR can justifiably be indicated for severe IMR. As for moderate IMR, CABG alone without mitral valve intervention may provide similar clinical outcomes.

Keywords: Ischemic mitral regurgitation (IMR); mitral valve replacement (MVR); cardiopulmonary bypass (CPB); aortic cross clamp; coronary artery bypass grafting (CABG)

Submitted Dec 14, 2017. Accepted for publication Apr 09, 2018.

doi: $10.21037 /$ jtd.2018.04.93

View this article at: http://dx.doi.org/10.21037/jtd.2018.04.93 


\section{Introduction}

Ischemic mitral regurgitation (IMR) is a common complication after myocardial infarction (MI). Approximately $50 \%$ of patients develop IMR after MI, and moderate or severe IMR occurs in more than $10 \%$ of them $(1,2)$. The presence of IMR predicts a poor prognosis. Previous studies have shown that greater severity of IMR indicates shorter long-term survival $(3,4)$. Even mild IMR increases mortality by $17 \%$ over that of patients without it (5).

The mechanisms of IMR are primarily associated with left ventricular remodeling after MI, which include papillary muscle displacement, leaflet tethering, reduced closing forces, and annular dilatation (6). Therefore, it is a subset of functional mitral regurgitation. The treatment of IMR has long been an issue of debate. This controversy is due to the lack of both clinical data and robust evidence. Recently, a practice guideline has identified severe IMR as a class I indication for surgical treatment (7). However, recommendations for the proper surgical modality, either repair or replacement, are still under investigation.

The optimal surgical treatment with regard to moderate IMR with concomitant coronary artery disease is also controversial. By conducting this retrospective study, we examined real-world surgical outcomes of patients with coronary artery disease and IMR managed by concomitant CABG and mitral valve operations in our hospital.

\section{Methods}

\section{Patient selection and data collection}

Between 2008 and 2015, 35 patients diagnosed with coronary artery disease with mitral regurgitation underwent CABG and mitral valve operations in our hospital. After the exclusion of patients whose etiologies of mitral regurgitation were not entirely ischemic, 22 patients remained in our series. The study was approved by the local ethics committee. The medical records for all patients were reviewed. The collected data included patient's pre-operative baseline characteristics, operation-related parameters, postoperative complications, hospitalization time, echocardiography data as ejection fraction and degree of residual mitral regurgitation, and mortalities. All patients had at least moderate mitral regurgitation, as determined by pre-operative transesophageal echocardiography (TEE). Residual mitral regurgitation was considered significant if a moderate or greater degree of mitral regurgitation was detected during follow-up echocardiography examination.
Four patients (18.2\%) were treated with MVR, and 18 patients $(81.8 \%)$, with mitral valve repair. Regarding the methods of mitral valve repair, three patients (16.7\%) received complete ring annuloplasty, and 15 patients $(83.3 \%)$ had simplified commissural annuloplasty.

The primary endpoints were early mortality, defined as any death occurring within 30 days of operation or before discharge from the hospital, and long-term survival.

\section{Operative techniques}

All surgeries were performed through midline sternotomy, with the use of cardiopulmonary bypass (CPB), mild systemic hypothermia $\left(32^{\circ} \mathrm{C}\right)$, and antegrade crystalloid cardioplegia. The decisions to perform repair or replacement were made at the discretion of individual surgeons. Concomitant coronary bypass surgery was performed on main coronary vessels or branches that displayed more than $70 \%$ luminal stenosis on a preoperative coronary angiogram.

\section{Statistical analysis}

Statistical analysis was performed in SPSS 22.0 (IBM, Armonk, NY, USA). Data are described as frequencies and means with standard deviations as appropriate. Pearson's chi-square was used for categorical variables, and Student's T-test was used for continuous variables. $\mathrm{P}$ values of $<0.05$ were considered statistically significant. A Kaplan-Meier survival curve was constructed.

\section{Results}

\section{Patient characteristics and operative data}

The baseline characteristics of the 22 patients (21 male; 1 female) are listed in Table 1 . The mean age was $62.1 \pm 11.4$ years. Eight patients $(36.4 \%)$ had atrial fibrillation before the operation. The mean left ventricular ejection fraction (LVEF) was $33.3 \pm 15.3$ preoperatively. The mean additive Euroscore was $7 \pm 3.7$, and the mean logistic Euroscore was $12.3 \% \pm 13.7 \%$.

The operative and postoperative details are provided in Table 2. The mean CPB time was $165.4 \pm 38.4$ minutes, and the mean aortic cross clamp time (AXC) was 113.8 33.6 minutes. The mean coronary bypass grafting number was $2.0 \pm 0.8$. Eleven patients $(50 \%)$ received intraaortic balloon pump (IABP) support after the operation. 
Table 1 Pre-operative baseline characteristics

\begin{tabular}{lc}
\hline Characteristics & Total (N=22) \\
\hline Age & $62.1 \pm 11.4$ \\
Gender (M/F) & $21 / 1$ \\
DM & $13(59.1)$ \\
HTN & $11(50.0)$ \\
Dyslipidemia & $7(31.8)$ \\
CKD (Cre >2) & $5(22.7)$ \\
Af & $8(36.4)$ \\
COPD & $3(13.6)$ \\
Old CVA & $1(4.5)$ \\
Pre-operative LVEF & $33.3 \pm 15.3$ \\
Euroscore (additive) & $7.0 \pm 3.7$ \\
Euroscore (\%) (logistic) & $12.3 \pm 13.7$ \\
\hline
\end{tabular}

M/F, male/female; DM, diabetes mellitus; HTN, hypertension; CKD, chronic kidney disease; Cre, creatinine; Af, atrial fibrillation; COPD, chronic obstructive pulmonary disease; CVA, cerebral vascular accident; LVEF, left ventricular ejection fraction.

Table 2 Operative and post-operative details

\begin{tabular}{|c|c|}
\hline Parameters & Total $(\mathrm{N}=22)$ \\
\hline CPB time (min) & $165.4 \pm 38.4$ \\
\hline AXC time (min) & $113.8 \pm 33.6$ \\
\hline Graft numbers & $2.0 \pm 0.8$ \\
\hline Postoperative IABP & $11(50.0)$ \\
\hline New-onset Af & $6(27.3)$ \\
\hline ARF requiring hemodialysis & $2(9.1)$ \\
\hline TND & $3(13.6)$ \\
\hline PND & $0(0)$ \\
\hline Post-operative ventilator (hrs) & $55.2 \pm 44.5$ \\
\hline Respiratory failure requiring tracheostomy & $2(9.1)$ \\
\hline LOIS (days) & $8.2 \pm 9.1$ \\
\hline LOHS (days) & $25.6 \pm 29.7$ \\
\hline Last LVEF & $39.9 \pm 16.3$ \\
\hline \multicolumn{2}{|c|}{$\begin{array}{l}\text { CPB, cardiopulmonary bypass; AXC, aortic cross clamp; IABP, } \\
\text { intra-aortic balloon pump; Af, atrial fibrillation; ARF, acute renal } \\
\text { failure; TND, transient neurologic dysfunction; PND, permanent } \\
\text { neurologic dysfunction; hrs, hours; LOIS, length of intensive } \\
\text { care unit stay; LOHS, length of hospital stay; LVEF, left } \\
\text { ventricular ejection fraction. }\end{array}$} \\
\hline
\end{tabular}

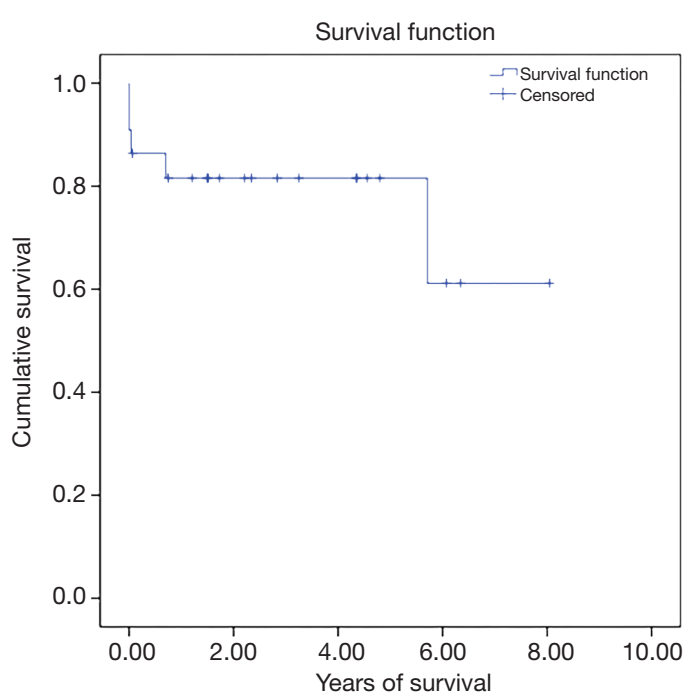

Figure 1 Kaplan-Meier survival curve of the whole group (mean follow-up duration: $3.1 \pm 2.3$ years).

Six patients $(27.3 \%)$ had new onset atrial fibrillation postoperatively. Two patients $(9.1 \%)$ required tracheostomy due to prolonged weaning from the ventilator. The mean LVEF during the last echocardiography follow-up was $39.9 \% \pm 16.3 \%$. The mean length of intensive care unit stay was $8.2 \pm 9.1$ days, and the mean length of hospital stay was $25.6 \pm 29.7$ days.

\section{Early mortality and long-term survival}

There were three in hospital mortalities (13.6\%) and two long-term mortalities. The 5-year long-term survival rate was $81.6 \%$. Figure 1 shows the Kaplan-Meier survival curve of the whole group. Among the three in-hospital mortalities, two died immediately after the operation due to intractable heart failure, and the other died of pneumonia and septic shock 2 weeks after the operation. With regard to the long-term mortalities, one had severe heart failure after the operation and prolonged hospitalization. He was found to have an out-of-hospital cardiac arrest 8 months after the operation. The other patient led an uneventful life after the operation. However, he died due to unrelated cancer issues 6 years later.

We divided the patients into a mitral valve repair group and a replacement group in order to examine if the two surgical modalities had any impact on early mortality and long-term survival. The baseline characteristics in both groups were statistically similar except in gender and chronic obstructive pulmonary disease prevalence (Table 3). 
Table 3 Pre-operative baseline characteristics (mitral valve repair vs. replacement)

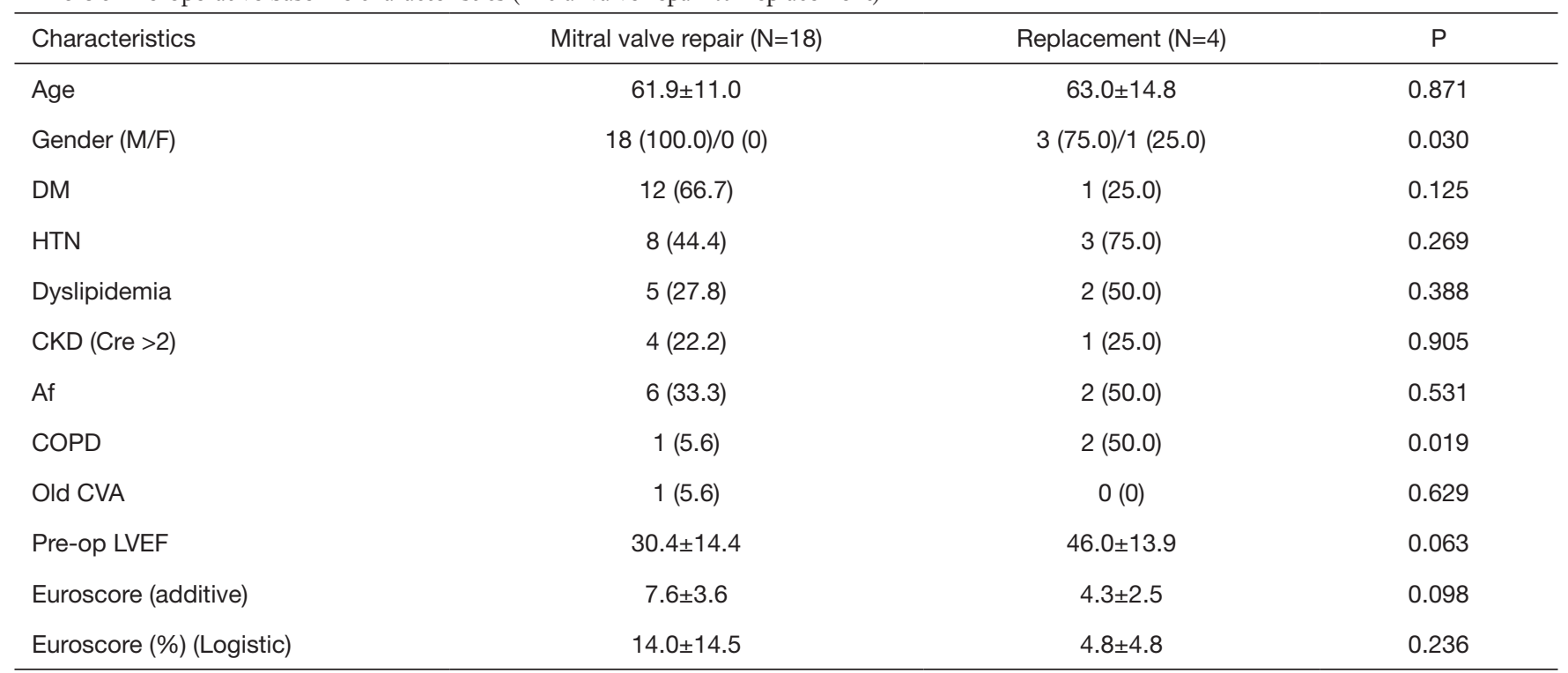

M/F, male/female; DM, diabetes mellitus; HTN, hypertension; CKD, chronic kidney disease; Cre, creatinine; Af, atrial fibrillation; COPD, chronic obstructive pulmonary disease; CVA, cerebral vascular accident; LVEF, left ventricular ejection fraction.

Table 4 Operative and post-operative details (mitral valve repair $v s$. replacement)

\begin{tabular}{|c|c|c|c|}
\hline Parameters & Mitral valve repair $(\mathrm{N}=18)$ & Replacement $(\mathrm{N}=4)$ & $\mathrm{P}$ \\
\hline AXC time (min) & $106.1 \pm 28.3$ & $148.5 \pm 58.7$ & 0.244 \\
\hline Graft numbers & $2.00 \pm 0.84$ & $1.75 \pm 0.96$ & 0.604 \\
\hline Postoperative IABP & $10(55.6)$ & $1(25.0)$ & 0.269 \\
\hline ARF requiring hemodialysis & $1(5.6)$ & $1(25.0)$ & 0.221 \\
\hline TND (ICU syndrome) & $2(11.1)$ & $1(25.0)$ & 0.464 \\
\hline PND (new CVA, hypoxia) & $0(0)$ & $0(0)$ & - \\
\hline Post-operative ventilator (hrs) & $50.6 \pm 38.1$ & $73.7 \pm 68.5$ & 0.365 \\
\hline LOHS (days) & $27.9 \pm 32.4$ & $15.8 \pm 10.9$ & 0.474 \\
\hline Last LVEF & $34.4 \pm 17.1$ & $38.5 \pm 22.6$ & 0.693 \\
\hline Early mortality & $2(11.1)$ & $1(25.0)$ & 0.464 \\
\hline
\end{tabular}

CPB, cardiopulmonary bypass; AXC, aortic cross clamp; IABP, intra-aortic balloon pump; Af, atrial fibrillation; ARF, acute renal failure; TND, transient neurologic dysfunction; PND, permanent neurologic dysfunction; hrs, hours; LOIS, length of intensive care unit stay; LOHS, length of hospital stay; LVEF, left ventricular ejection fraction. 


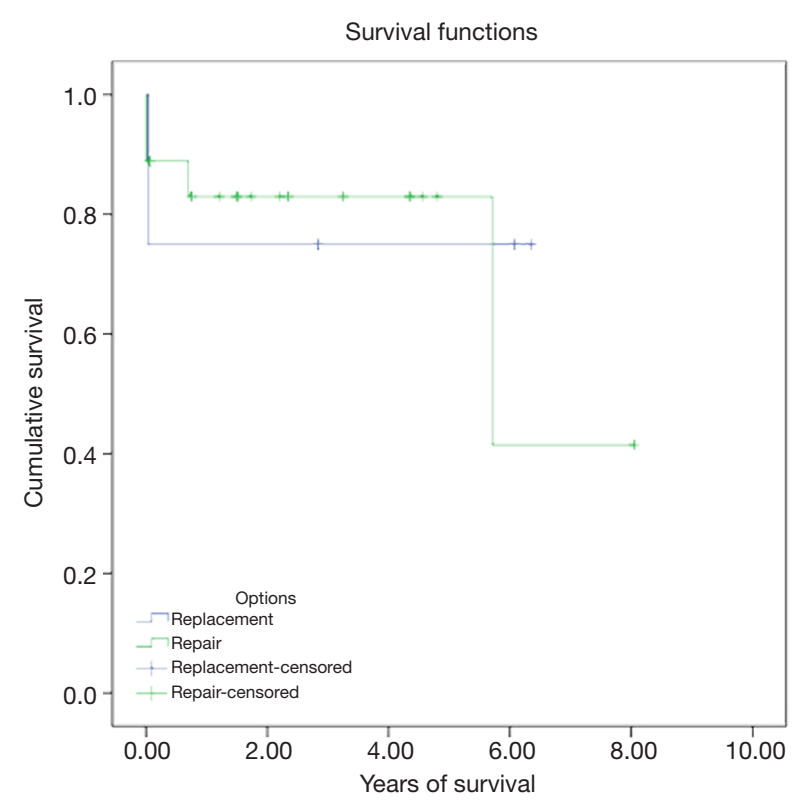

Figure 2 Kaplan-Meier survival curve (mitral valve repair vs. replacement) (mean follow-up duration: $3.1 \pm 2.3$ years) $(\mathrm{P}=0.801)$.

However, our results did not reach statistical significance for either parameter [early mortality, $11.1 \%(2 / 18)$ in repair group vs. $25.0 \%$ (1/4) in replacement group, $\mathrm{P}=0.464$; 5 -year long-term survival, $83.0 \%$ in repair group vs. $75.0 \%$ in replacement group, Kaplan-Meier survival curve log rank (Mantel-Cox) $\left.x^{2}=0.06, \mathrm{P}=0.801\right]$ (Table 4 and Figure 2).

\section{Residual or recurrent mitral regurgitation}

Among the seventeen survivors, three had MVR and fourteen had mitral valve repair. All patients with MVR survived for more than 3 years. At their last echocardiography follow-up, either no or trivial mitral regurgitation was found. Therefore, the residual or recurrent mitral regurgitation rate, defined as more than a moderate degree by echocardiography examination, was $0.0 \%$.

In the mitral repair group, three patients had complete ring annuloplasty, and the rest received simplified commissural annuloplasty. Considering that a short interval between operation and follow-up echocardiography examination may not reveal the development of recurrent mitral regurgitation, we limited the analysis to patients with data available for more than or equal to 1 year after the operation. Seven patients were found to fall within this subgroup. Four of them had at least moderate mitral regurgitation during their last echocardiography follow-up.
The residual or recurrent mitral regurgitation rate was $57.1 \%$ (4/7). If we subdivide these seven patients by repair methods, one was from the ring annuloplasty group and six were from the commissural annuloplasty group. The residual or recurrent mitral regurgitation rates were $100.0 \%(1 / 1)$ in the ring annuloplasty group and 50.0\% (3/6) in the commissural annuloplasty group. Moreover, one patient, whose repair was performed with simplified commissural annuloplasty, underwent a subsequent reoperation for MVR two years after the initial repair due to severe regurgitation. Table 5 presents the echocardiography details among the 17 survivors.

\section{Discussion}

In our series, the early mortality and late survival rates of mitral valve repair were similar to those of MVR. This finding might imply a similar role of the two treatment options. However, numerous reports have shown that the ingenuity of the disease itself renders the optimal management a complex issue (8-10), so the optimal management approach is still controversial. Practice among cardiac surgeons varies greatly regarding IMR treatment, mostly due to a lack of data on this issue. Therefore, as in our institute, most cardiac surgeons may make surgical decisions according to their own personal beliefs and experiences. To the best of our knowledge, no guideline for cardiac surgeons to follow on this topic has been established.

In order to simplify this complex disease scenario, it is reasonable to consider different treatment options according to the severity of mitral regurgitation. For severe IMR, the general consensus is that surgical treatment is indicated (7). However, recommendations for either mitral valve repair or replacement remain less definitive. A recent randomized controlled trial (RCT) by Acker et al. (8) compared mitral valve repair with chordal-sparing MVR in patients with severe IMR. Their study revealed no significant difference in left ventricular reverse remodeling or survival at 1 year follow-up, while replacement provided a more durable correction of mitral regurgitation. This finding was reflected in our series as well, with a residual or recurrent mitral regurgitation rate of $57.1 \%(4 / 7)$ in the mitral valve repair group, as compared to a rate of $0.0 \%$ in the replacement group. It also held true when we subdivided patients in the mitral repair group into the ring annuloplasty group $(100.0 \% ; 1 / 1)$ and the commissural annuloplasty group $(50.0 \% ; 3 / 6)$ (Table 5$)$. The trial cohort was then followed, and the result remained the same at 
Table 5 Echocardiography details among 17 survivors

\begin{tabular}{|c|c|c|c|c|c|c|c|c|c|}
\hline Patient Number & $\begin{array}{l}\text { Pre-op TEE } \\
\text { MR grade }\end{array}$ & $\mathrm{EF}(\%)$ & $\begin{array}{c}\text { SPAP } \\
(\mathrm{mmHg})\end{array}$ & $\begin{array}{l}\text { LVESVI } \\
\left(\mathrm{mL} / \mathrm{m}^{2}\right)\end{array}$ & $\begin{array}{l}\text { Last echo MR } \\
\text { grade }\end{array}$ & EF (\%) & $\begin{array}{c}\text { SPAP } \\
(\mathrm{mmHg})\end{array}$ & $\begin{array}{l}\text { LVESVI } \\
\left(\mathrm{mL} / \mathrm{m}^{2}\right)\end{array}$ & $\begin{array}{l}\text { Interval between } \\
\text { op and last echo }\end{array}$ \\
\hline \multicolumn{10}{|l|}{ MV replacement } \\
\hline No. 2 & Mod-severe & 45 & N/A & 45.60 & Trivial & 16 & 41 & 109.33 & $6 \mathrm{yr}$ \\
\hline No. 4 & Severe & 16 & 65 & 70.48 & Mod & 19 & 72 & 76.51 & $2 \mathrm{yr}$ \\
\hline No. 5 & Mod & 35 & N/A & 72.47 & Mild & 53 & N/A & 31.46 & $1 \mathrm{mo}$ \\
\hline No. 6 & Mod-severe & 31 & 50 & 77.37 & Mod & 25 & 63 & 81.58 & $9 \mathrm{mo}$ \\
\hline No. 9 & Mod & 25 & 45 & 62.38 & Mod-severe & 25 & 60 & 107.92 & $5 \mathrm{yr}$ \\
\hline No. 10 & Mod & 20 & 39 & 57.46 & Mod & 26 & 19 & 65.75 & $4 \mathrm{yr}$ \\
\hline No. 11 & Mod-severe & 33 & 58 & 46.20 & Mild & 64 & 28 & 23.42 & $2 \mathrm{yr}$ \\
\hline No. $12^{*}$ & Mod-severe & 30 & 74 & 70.48 & Severe & 41 & 88 & 56.63 & $2 \mathrm{yr}$ \\
\hline No. 13 & Mod-severe & 31 & 56 & 91.49 & Mild & 18 & $N / A$ & 97.34 & $1 \mathrm{yr}$ \\
\hline No. 14 & Mod & 65 & $\mathrm{~N} / \mathrm{A}$ & 21.47 & Trivial & 63 & N/A & 13.56 & $7 \mathrm{mo}$ \\
\hline No. 15 & Mod-severe & 20 & 57 & 102.31 & Mild-Mod & 22 & 39 & 97.69 & $2 w k$ \\
\hline
\end{tabular}

*, this patient had subsequent mitral valve replacement due to last echocardiography finding of severe mitral regurgitation. Op, operation; TEE, trans-esophageal echocardiography; MR, mitral regurgitation; EF, ejection fraction; SPAP, systolic pulmonary artery pressure; LVESVI, left ventricular end-systolic volume index (= left ventricular end-systolic volume/body surface area); echo, echocardiography; Mod, moderate; N/A, not applicable; yr, year; MV, mitral valve; mo, month; wk, week.

2 years (9). In their study, patients who underwent mitral valve repair had more frequent recurrence of mitral regurgitation, resulting in more heart-failure related adverse events and readmissions.

Although within the trial cohort, the survival was compatible in both groups, a large meta-analysis report regarding this issue found a different result (10). A metaanalysis by Virk et al. included twenty-two observational retrospective studies and one RCT. The retrospective studies showed better long-term survival in the mitral valve repair group. We found that most of the studies were followed for more than 3 years. Therefore, it can be speculated that the effect on survival may require a longer follow-up duration to become evident. In our series, the mean follow-up duration was more than 3 years. The 5 -year survival of the mitral valve repair group was better than that of the replacement group (83.0\% vs. $75.0 \%)$. However, the difference did not reach statistical significance, possibly due to our small sample size. A longer follow-up on the RCT in the future is warranted to detect any difference in survival.

In contrast, for patients with moderate IMR, the issue of whether mitral valve repair is necessary in addition to CABG is still controversial. Proponents of adding mitral valve repair to CABG argue that $40 \%$ of patients continue to have moderate or severe mitral regurgitation after isolated CABG and that persistent regurgitation may lead 
to worse outcomes (11). Some studies have suggested a functional benefit from concomitant mitral valve surgery, while others have found neither symptomatic nor survival benefits from the combination of mitral valve surgery and coronary artery bypass surgery.

Smith et al. conducted the largest RCT on this topic (12). Their study showed that combining mitral valve repair with $\mathrm{CABG}$ was associated with a reduced prevalence of moderate or severe mitral regurgitation but an increased number of untoward events. Although mortality and major adverse cardiac or cerebrovascular event rates did not differ significantly between the two groups, the neurologic event rate and supraventricular arrhythmia rate were higher in the mitral valve repair group. This difference was believed to be related to the required longer $\mathrm{CPB}$ time and obligatory atriotomy incision, which is necessary for mitral valve repair to be performed. The follow-up of that trial demonstrated similar results at 2 years (13). The data revealed certain risks of adverse perioperative events due to additional mitral valve repair. However, with the advent of the percutaneous mitral valve repair technique, this risk may be reduced and the benefit of mitral valve repair retained (14).

Surgeons advocating isolated CABG argue that treating the underlying cause leads to reverse remodeling of the left ventricle, which in turn decreases mitral regurgitation. The success of such an approach relies greatly on the presence of viable myocardium. Penicka et al. (15) indicated that improvement in regurgitation in patients with moderate IMR who underwent isolated CABG was limited to those who had viable myocardium and an absence of papillary muscle dyssynchrony.

Viable myocardium plays a pivotal role in the efficacy of surgical revascularization. Successful revascularization is favorable to mitral valve function in patients with IMR, which is related to a decrease in left ventricular size, increased mitral valve closing force, improved papillary synchrony, and enhanced myocardial contractility (13). In our practice, we prefer to perform viability tests by thallium scan, cardiac magnetic resonance imaging, or positron emission tomography prior to the operation. We believe that precise revascularization of the target coronary vessels with viable myocardium is beneficial to the clinical outcome, reduces the degree of IMR severity, and obviates the need to address the IMR issue.

Castleberry et al. (16) also supported this concept by publishing the largest real world dataset to date. It was a single-center retrospective analysis. They examined 4,989 patients with moderate or severe IMR over a
10 -year period. Patients in this study were managed with medication, percutaneous coronary intervention, CABG, or concomitant $\mathrm{CABG}$ and mitral valve surgery. Among these treatments, isolated CABG achieved the highest adjusted survival at 10 years. A meta-analysis by Kopjar et al. (11), which included 5 observational studies and 4 RCTs, also concluded that, for moderate IMR, neither increased operative mortality nor survival benefit was associated with concomitant CABG and mitral valve repair over CABG alone.

Even though the evidence from the above studies suggests that isolated CABG can provide compatible clinical outcomes and survival, it is also clear that CABG combined with mitral valve repair can be performed safely, and that the combined procedure may be advantageous as compared to isolated $\mathrm{CABG}$ in a certain subset of patients due to the theoretical benefit of eliminating mitral regurgitation and its associated adverse impact on left ventricular remodeling. Therefore, a future study should be directed to defining which patients may benefit the most from the concomitant mitral valve operation for moderate IMR during coronary bypass surgery.

To summarize the above discussion, the severity of mitral regurgitation might be the critical clinical element that can help us to decide which surgical modality to apply for IMR patients. Based on the two important RCT studies $(8,12)$, we suggest that severe IMR patients should undergo MVR. The underlying possible mechanism behind this recommendation is that MVR provides more durable correction than does mitral valve repair, while it maintains similar clinical survival to that of mitral valve repair. On the other hand, we suggest that moderate IMR patients undergo isolated CABG as long as there is viable myocardium supplied by target vessels to be revascularized. Therefore, the myocardium viability test could be another important clinical element for the decision making in patients of moderate IMR. The possible mechanism for isolated $\mathrm{CABG}$ instead of CABG plus mitral valve repair is that the addition of mitral valve repair carries elevated risks of neurological events and supraventricular arrhythmias, while it provides no survival advantages compared to CABG alone, as noted in Smith's study (12). We suggest that if there is no viable myocardium supplied by target vessels to be revascularized, a deliberate mitral valve repair may then become necessary. The aforementioned mechanisms need to be validated. We hope that after confirming these mechanisms, the outcome of surgical management for IMR patients can be improved and ultimately the related methods can be extended to other centers. 


\section{Limitations}

This study has four limitations. First of all, as a retrospective observational study, it is subject to inherent bias. Second, our study was limited by the small sample size, which can explain the lack of statistical significance among our results. A future study with multi-institution collaboration would provide large patient numbers/data and robust results. Third, the grading of mitral regurgitation may not have been accurate due to individual operators' judgements and patients' volume status during the assessment. Fourth, we did not collect data of another subgroup of IMR patients who underwent isolated CABG only, so we could not compare the effect of isolated CABG to CABG plus mitral valve operations.

\section{Conclusions}

Mitral valve repair might be comparable to MVR in our series in terms of early mortality and long-term survival. However, mitral valve repair portended a higher risk of residual or recurrent mitral regurgitation one year after operation. The optimal surgical strategy is controversial due to the paucity of clinical data. According to the current literature, the role of MVR seems to be justified in patients with severe IMR. In patients with moderate IMR, isolated CABG may offer clinical outcomes similar to those of the combination of mitral valve repair and coronary artery bypass surgery.

\section{Acknowledgements}

None

\section{Footnotes}

Conflicts of Interest: The authors have no conflicts of interest to declare.

Ethical Statement: The study was approved by the institutional review board of the hospital (Number of the Ethical Approval: CGH-P106069).

\section{References}

1. Bursi F, Enriquez-Sarano M, Nkomo VT, et al. Heart failure and death after myocardial infarction in the community: the emerging role of mitral regurgitation.
Circulation 2005;111:295-301.

2. Perez de Isla L, Zamorano J, Quezada M, et al. Prognostic significance of functional mitral regurgitation after a first non-ST-segment elevation acute coronary syndrome. Eur Heart J 2006;27:2655-60.

3. Grigioni F, Enriquez-Sarano M, Zehr KJ, et al. Ischemic mitral regurgitation: long-term outcome and prognostic implications with quantitative Doppler assessment. Circulation 2001;103:1759-64.

4. Bursi F, Enriquez-Sarano M, Jacobsen SJ, et al. Mitral regurgitation after myocardial infarction: a review. Am J Med 2006;119:103-12.

5. Lamas GA, Mitchell GF, Flaker GC, et al. Clinical significance of mitral regurgitation after acute myocardial infarction. Survival and Ventricular Enlargement Investigators. Circulation 1997;96:827-33.

6. Kono T, Sabbah HN, Rosman H, et al. Left ventricular shape is the primary determinant of functional mitral regurgitation in heart failure. J Am Coll Cardiol 1992;20:1594-8.

7. Nishimura RA, Otto CM, Bonow RO, et al. 2014 AHA/ ACC guideline for the management of patients with valvular heart disease: a report of the American College of Cardiology/American Heart Association Task Force on Practice Guidelines. Circulation 2014;129:e521-643.

8. Acker MA, Parides MK, Perrault LP, et al. Mitral-valve repair versus replacement for severe ischemic mitral regurgitation. N Engl J Med 2014;370:23-32.

9. Goldstein D, Moskowitz AJ, Gelijns AC, et al. Two-year outcomes of surgical treatment of severe ischemic mitral regurgitation. N Engl J Med 2016;374:344-53.

10. Virk SA, Sriravindrarajah A, Dunn D, et al. A metaanalysis of mitral valve repair versus replacement for ischemic mitral regurgitation. Ann Cardiothorac Surg 2015;4:400-10.

11. Kopjar T, Gasparovic H, Mestres CA, et al. Metaanalysis of concomitant mitral valve repair and coronary artery bypass surgery versus isolated coronary artery bypass surgery in patients with moderate ischemic mitral regurgitation. Eur J Cardiothorac Surg 2016;50:212-22.

12. Smith PK, Puskas JD, Ascheim DD, et al. Surgical treatment of moderate ischemic mitral regurgitation. $\mathrm{N}$ Engl J Med 2014;371:2178-88.

13. Michler RE, Smith PK, Parides MK, et al. Two-year outcomes of surgical treatment of moderate ischemic mitral regurgitation. N Engl J Med 2016;374:1932-41.

14. Scoville DH, Boyd JB. A novel approach to ischemic mitral regurgitation (IMR). Ann Cardiothorac Surg 
2015;4:443-8.

15. Penicka M, Linkova H, Lang O, et al. Predictors of improvement of unrepaired moderate ischemic mitral regurgitation in patients undergoing elective isolated coronary artery bypass graft surgery. Circulation
2009;120:1474-81.

16. Castleberry AW, Williams JB, Daneshmand MA, et al. Surgical revascularization is associated with maximal survival in patients with ischemic mitral regurgitation: a 20-year experience. Circulation 2014;129:2547-56.
Cite this article as: Lee ML, Chen TH, Huang HD, Hou SM. Mitral valve repair versus replacement in patients with ischemic mitral regurgitation. J Thorac Dis 2018;10(5):2820-2828. doi: 10.21037/jtd.2018.04.93 